\title{
Le document de presse dans un cours de FLE/FOS : de la théorie à la pratique de classe
}

\author{
Nuria RODRÍGUEZ PEDREIRA \\ Universidad de Santiago de Compostela \\ nuria.rodriguez@usc.es
}

Recibido: $14 / 10 / 2011$

Aceptado: 14/11/2012

\begin{abstract}
Résumé
Après un survol des caractéristiques liées aux stratégies d'apprentissage et aux compétences à acquérir en langue cible, nous présentons une démarche de lecture, issue d'une Approche Globale (AG), appliquée à un texte de divulgation extrait de la presse numérique. Ainsi, les activités proposées sont centrées sur les stratégies de lecture conduisant à l'acquisition de la compréhension écrite, et à une plus grande autonomie de l'étudiant dans la maîtrise de cette compétence. La procédure peut s'élargir à d'autres domaines d'enseignement, et peut s'avérer utile aux enseignants comme aux apprenants. Aussi, les premiers pourront-ils modifier et adapter les activités en fonction du public cible (son niveau de langue, ses objectifs, ses intérêts), tandis que les seconds pourront devenir plus autonomes grâce à l'appropriation d'une bonne compétence de lecture, et appliquer la démarche à d'autres types de supports écrits.
\end{abstract}

Mots clés: didactique des langues, FLE, FOS, stratégies de lecture, compréhension écrite.

\section{El documento de prensa en un curso de FLE/FOS: de la teoría a la práctica de clase}

\section{Resumen}

Tras un breve repaso de los rasgos asociados a las estrategias de aprendizaje y a las competencias que hay que alcanzar en la lengua meta, presentamos un modelo de lectura, basado en una Metodología Global, aplicado a un texto de divulgación extraído de la prensa digital. Así, las actividades propuestas se centran en las estrategias de lectura conducentes a la adquisición de la comprensión escrita y a una mayor autonomía del estudiante en el dominio de esta competencia. Nuestra propuesta es flexible por ser extrapolable a otros ámbitos de la enseñanza, y puede resultar útil tanto a enseñantes como a aprendices. Los primeros podrán modificar y adaptar las actividades en función del público asistente (su nivel de lengua, sus objetivos e intereses), mientras que los segundos podrán alcanzar mayor autonomía gracias a la adquisición de una buena competencia de lectura, pudiendo ambos aplicar las estrategias propuestas a otros tipos de soportes escritos.

Palabras clave: didáctica de lenguas, FLE, FOS, estrategias de lectura, comprensión escrita. 


\title{
The press article in an FFL/FSP course: from theory to practice
}

\begin{abstract}
Starting from a review of the features connected with learning strategies and with the competencies to develop in the target language, we apply reading procedures based on a Global Approach to a text extracted from the digital press. The pedagogical implementations shown are focused on reading techniques that guarantee a correct comprehension and a greater autonomy of the student in the acquisition of this skill. The strategies presented can be applied to other fields of education, and are valid as much for teachers as for students. Instructors will be able to modify and adapt the activities according to their audiences (level of language, objectives, interests...), whereas students will be able to become more independent after they improve their reading competence, which will then be applicable to texts in other formats and media.
\end{abstract}

Key words: language pedagogy, FFL, FSP, reading strategies, written comprehension.

\section{Referencia normalizada}

Rodríguez Pedreira, N. (2013). "Le document de presse dans un cours de FLE/FOS : de la théorie à la pratique de classe". Thélème, Vol. 28, 221-242.

Sumario: 1. Introduction. 2. Les stratégies d'apprentissage. 2.1. Les stratégies de lecture. 3. Les compétences visées. 3.1. Compétences générales 3.2. Compétences linguistiques 3.3. Compétences pragmatiques. 4.1. Les activités de réception. 4. Démarche de lecture d'un texte de presse: les activités de classe. 5. En guise de conclusion.

\section{Introduction}

En vue de fournir aux enseignants des pistes de réflexion pour l'exploitation d'un texte de presse dans un cours de langue étrangère, cette étude présente un certain nombre d'activités conçues à des fins didactiques. Sans qu'on puisse parler pour autant de proposition pédagogique à part entière, les tâches présentées constituent un ensemble varié de questions, de suggestions, de stratégies élémentaires, que les enseignants pourront, en partie ou en totalité, appliquer durant les séances de cours. On s'efforcera, au passage, de faire ressortir l'intérêt de l'Approche Globale (AG) de la lecture, en dépit des positionnements critiques avec certaines de ses notions ${ }^{1}$.

${ }^{1}$ Rui (2000) passe au crible de l'examen critique le concept de stratégie de lecture, rattaché à l'AG. Selon l'auteur, il constituerait « uniquement une réflexion à visée pédagogique à partir d'un point de vue d'enseignement; que, de ce fait, elle ne théorise pas les opérations effectuées par le lecteur » (Rui, 2000:3). Quoi qu'il en soit, la notion marque bel et bien une rupture avec les postulats théoriques traditionnels de didactique de l'écrit. 
Destinées à des apprenants espagnols de différents niveaux et contextes, universitaires ou adultes, les activités suggérées peuvent être exploitées autant dans le domaine du français langue étrangère (FLE) que dans celui du français sur objectifs spécifiques (FOS). Tant et si bien que la thématique et le contenu du document en ligne sélectionné le rendent, à toutes fins, exploitable dans une Faculté de Lettres, dans une École de Commerce comme dans un Centre de Langues. De plus, optant pour le journal numérique comme voie d'exploitation rapide et efficace, nous intégrons les TICE (technologies de l'information et de la communication dans l'enseignement) à l'apprentissage, en redoublant la motivation et l'intérêt pour la langue étrangère apprise, le français dans le cas qui nous occupe.

Dans la dernière décennie, les TICE n'ont cessé de jouer un rôle croissant tant dans le processus de l'enseignement que dans celui de l'apprentissage.

Pour le FLE et le FOS, les méthodes récentes témoignent de cet état de fait. Non seulement sont-elles centrées sur l'approche actionnelle et tournées vers l'accomplissement de tâches ${ }^{2}$, mais en plus possèdent-elles un matériel audio (CDRom, DVD, fichiers mp3) et des éditions numériques préparées pour les TBI (Tableau blanc interactif). Outre les méthodes en support papier ou numériques, le réseau Internet constitue de nos jours une excellente source d'information dans laquelle puiser des textes électroniques en ligne, sans limite de temps ni d'espace. Ces documents deviennent un support précieux pour enseignants et étudiants, qui disposent de ressources variées à portée de main, toujours actualisées, libres d'accès et foisonnant de potentialités didactiques. C'est ce qui apparaît clairement, par exemple, dans les lignes suivantes :

L'avènement du multimédia offre de nombreuses possibilités didactiques. Dans la mise en place de programmes FOS, qui impliquent des réponses individuelles, chaque fois différentes [...], le multimédia constitue un outil précieux. L'enseignant de FOS [...] trouvera sur Internet une documentation variée, souvent fiable (à condition de bien vérifier les sources), toujours actualisée. Certains supports peuvent ainsi être obtenus avec moins de difficultés et moins de temps (Carras et al., $2007: 34-35)$.

\footnotetext{
${ }^{2}$ Le concept de tâche étant défini dans le Cadre européen Commun de Référence pour les langues (CECR) comme : «l'un des faits courants de la vie quotidienne dans les domaines personnel, public, éducationnel et professionnel. L'exécution d'une tâche par un individu suppose la mise en œuvre stratégique de compétences données, afin de mener à bien un ensemble d'actions finalisées dans un certain domaine avec un but défini et un produit particulier » (Conseil de l'Europe, $2001: 121$ ). Bien que la définition reste trop générale, celle-ci peut être nuancée lorsqu'elle s'applique à l'enseignement et à l'apprentissage d'une langue. Ainsi, dans une tâche communicative, non seulement l'attention doit-elle être focalisée sur des activités incluant plusieurs compétences linguistiques, mais aussi sur la signification que la tâche doit avoir pour l'apprenant, et sur sa motivation à l'exécuter correctement.
} 
Au-delà des activités proprement actionnelles ${ }^{3}$, particulièrement adaptées aux objectifs de FOS — puisqu'elles permettent aux apprenants de mettre à l'œuvre leurs compétences langagières en situation professionnelle-, la presse constitue un bon moyen de se tenir au courant de l'actualité politique, économique, sociale et culturelle en France et dans le monde. Ainsi, les internautes disposent-ils de dossiers en ligne leur permettant de cibler un sujet d'actualité dans ses différents domaines (politique, tourisme, économie, société, sciences...), ressources qui s'avèrent très utiles aux enseignants et aux apprenants. L'accès aux sites Internet des différents médias français peut donc être exploité à des fins pédagogiques, tout en permettant aux internautes de suivre l'actualité de la France dans ses différentes disciplines. Les documents qui figurent sur ces portails sont de nature très variée, pouvant aller du document texte au document audio, ou même à la vidéo.

Pour notre part, nous allons focaliser notre attention sur un document texte authentique ${ }^{4}$ relevant du domaine économique. La source consultée est lepetitjournal.com, le Journal des Français à l'étranger et des francophones. Plusieurs raisons ont guidé notre choix. D'une part, les articles paraissant sur ce site sont courts, clairs et concis, ce qui rend l'exploitation pédagogique utile à plusieurs niveaux. D'autre part, il s'agit d'une ressource en ligne permettant de véhiculer et de transmettre des contenus d'information, mais également des contenus d'enseignement. Et c'est précisément dans ce dernier point que l'enseignant doit intervenir « lors de l'étape de didactisation des documents retenus » (Carras et al., $2007: 34$ ), en proposant des activités adaptées au niveau des apprenants, tel qu'il apparaît dans les propos suivants:

Il faut tout d'abord adapter les données recueillies au niveau des apprenants. Cela peut revenir à simplifier un document, ou à choisir de ne travailler que certains points très précis. Il faut également sélectionner les points à travailler en fonction des objectifs des apprenants (Carras et al., $2007: 34)$.

${ }^{3}$ Celles qui font agir et réagir les apprenants dans une situation réelle de communication linguistique, en fournissant soit des productions orales (jeux de rôle ou simulations globales), soit des productions écrites (rallyes Internet, par exemple).

${ }^{4}$ Un document dit authentique n'a pas été conçu pour être utilisé en classe, contrairement aux outils fabriqués. Il s'agit à l'origine d'un outil sans caractère pédagogique. Dans une étude interrogeant la notion d'authenticité à la lumière de la situation des migrants en apprentissage, Adami (2009) conteste la dichotomie document authentique vs document fabriqué, et ne parle que de continuum: "Nous l'avons vu, l'authenticité parfaite est une illusion dans la mesure où un document est forcément didactisé, à partir du moment où il est utilisé dans une situation d'apprentissage guidé. Ceci étant, les documents utilisés peuvent être plus ou moins proches de la réalité. Je ne vois ainsi pas d'opposition dichotomique entre documents authentiques et documents fabriqués mais un continuum. Le seul véritable document authentique ne serait précisément pas un «document» mais les interactions verbales orales et écrites produites en situation naturelle » (Adami, $2009: 167$ ). Nous tenons à préciser que la dénomination authentique comporte pour nous cette part de didactisation qui va de soi. Transformé en un outil de classe, le document est forcément extrait de son contexte communicationnel d'origine. 
Les exercices, les tâches et d'autres ressources utilisées à des fins didactiques (les scénarios pédagogiques, entre autres) ${ }^{5}$ doivent viser des objectifs d'apprentissage et travailler des compétences précises. Avant de suggérer des pistes d'exploitation pédagogique du document de presse sélectionné (\& 4.), rappelons quelques traits relatifs aux stratégies d'apprentissage (\& 2.) et aux compétences visées (\& 3.).

\section{Les stratégies d'apprentissage}

Les didacticiens s'accordent, en général, pour admettre que le rôle de l'enseignant doit faire de nos jours plus figure de médiateur, d'animateur de classe, que de simple transmetteur de connaissances. C'est dire que l'enseignement/apprentissage tend vers l'autonomisation, et que les stratégies d'apprentissage apparaissent comme un moyen efficace d'acquérir une compétence professionnelle de façon autonome. Cette nécessité est signalée dans les propos qui suivent :

L'enseignant s'efforcera d'autonomiser les apprenants, et de leur fournir des stratégies d'apprentissage [...]. Il importe donc de donner aux apprenants des stratégies d'apprentissage qui leur permettent de s'autoformer, d'être autonomes dans leur apprentissage (Carras et al., 2007 : 40).

L'acquisition de cette compétence ne deviendra possible qu'à travers des outils linguistiques susceptibles d'être utilisés dans une classe de FLE/FOS ${ }^{6}$, comme les activités de production et de réception. Il importe également de se demander de quelle façon et en quel ordre le déroulement de ces activités pourra être mené. Outre le fait que l'organisation des contenus d'un cours dépend, en partie, du but à atteindre et des connaissances à acquérir durant la période d'enseignement, la planification des tâches et le choix des priorités de la part de l'enseignant s'avèrent indispen-

\footnotetext{
${ }^{5}$ Les scénarios pédagogiques sont utilisés par l'enseignant pour l'apprentissage de ses étudiants. Il s'agit d'activités basées sur une feuille de route contenant des indications à suivre et les objectifs pédagogiques à atteindre. Celles-ci offrent pour beaucoup une forte motivation, puisque l'utilisation des ressources de l'Internet ou multimédia s'avère indispensable.

${ }^{6}$ Là où un programme FLE se situe dans une approche globale de la langue, et traite une diversité de thèmes, le programme FOS répond à des besoins précis de formation, manifestés par un publiccible spécifique, et à une démarche d'enseignement centrée sur des situations de communication particulières. Mais pour les deux, l'objectif est de communiquer avec fluidité en français. Compte-tenu de sa spécificité, le document choisi à des fins didactiques est destiné, en premier lieu, à des étudiants espagnols de FOS qui nécessitent une formation de français dans leur domaine de spécialité (ADE (Administración y Dirección de empresas), Faculté des sciences économiques, etc.). Cependant, il pourrait se révéler tout aussi profitable à des apprenants espagnols de FLE, faux débutants ou ayant déjà acquis un certain niveau de langue, comme moyen de les motiver, et de les faire participer aux différentes activités de classe. Les étudiants de Philologie ou d'autres facultés de langues, par exemple, pourraient être concernés.
} 
sables pour l'acquisition d'un bon niveau de compétence en langue étrangère. Dans le cadre de ce travail, ce sont les stratégies de lecture qui retiendront notre attention.

\subsection{Les stratégies de lecture}

Sur la base d'une étude expérimentale de lecture de textes en L2 (langue seconde), menée au Département de langues, linguistique et traduction de l'Université Laval, avec «[...] plusieurs cohortes d'étudiants» (Boucher, $2002: 58$ ), Boucher précise que l'utilisation des textes de différente nature vise « [...] à faire de l'apprenant un lecteur à la fois autonome et efficace » (Boucher, 2002: 58):

Ils [textes courants] font notamment appel à un lexique souvent spécialisé et sont régis par des structures particulières, loin de celles de la narration littéraire. Cette spécificité du discours à caractère plus «scientifique» rend la lecture de ces textes particulièrement difficile pour les apprenants d'une langue seconde (Boucher, 2002 : 58).

La démarche de lecture proposée par Boucher vise non seulement l'autonomie des apprenants, mais aussi leur aptitude à identifier globalement la structure d'un texte, en en repérant les principaux axes autour desquels elle s'articule. Il s'agirait de se soustraire à une lecture linéaire qui finit souvent par frustrer les étudiants, qui perçoivent la lecture «comme un mal nécessaire» (Boucher, 2002: 59). Cette approche globale de la lecture n'est pas nouvelle, tant s'en faut. Vers les années 70, sous l'influence de la psychologie cognitive, la compréhension de l'écrit acquiert une nouvelle dimension axée sur les stratégies de réception. L'approche traditionnelle faisant place à une conception différente de la lecture : les étudiants observent le texte, ils en repèrent les éléments clés, puis tentent de contourner les zones opaques en partant de leurs connaissances et de leur vécu pour parvenir à la construction de la compréhension (Moirand, 1979; Lehmann \& Moirand, 1980 ; Cicurel, 1991). En d'autres termes, la lecture globale permettrait de saisir plus efficacement le sens du texte écrit. Le CECR propose, à ce sujet, un schéma qui pourrait favoriser la représentation $d u$ sens (Conseil de l'Europe, 2001: 59) ${ }^{7}$. Les conditions de réception obtenues par cette procédure permettraient d'orienter le lecteur sur l'intention communicative, et de parvenir à déchiffrer le message. En sachant bien que l'efficacité du processus dépend, selon nous, de la plus ou moins grande complexité du texte à lire, de sa thématique plus ou moins familière, et, bien entendu, du niveau de langue des étudiants, ainsi que de leurs capacités, linguistiques et non linguistiques pour mener à bien les différentes étapes d'activité réceptive.

C'est dans le cadre de l'AG que nous souhaitons aborder le présent travail, et présenter notre modèle didactique. Avant d'aller plus avant, rappelons, à la suite de

${ }^{7}$ La procédure déclenchée, lors des opérations de réception, est structurée en quatre étapes : planification, exécution, évaluation et remédiation. Pour un approfondissement sur la question, voir le CECR (Conseil de l'Europe, 2001 : 57-60). 
Grellet (1981: 4), les stratégies qui nous semblent devoir être mises en place pour l'acquisition d'une compétence de lecture efficace ${ }^{8}$ :

(a) La lecture écrémage (Skimming). L'accent est mis sur la compréhension globale. Il s'agit de repérer les mots-clés à l'aide des titres, intertitres, des marques typographiques ou iconiques, des photos. Cette démarche est particulièrement adaptée aux articles de presse, dans lesquels l'essentiel est dit dès les premières lignes. Le lecteur peut émettre des hypothèses sur le contenu, et parvenir à la construction de la compréhension.

(b) La lecture balayage ou ciblée (Scanning). Elle se focalise sur une information précise pour laquelle doivent être établis, a priori, les éléments à rechercher. Il s'agit d'une tâche plus complexe —et bien plus lourde aussi- qui nécessite d'un bon niveau de langue, et d'un entraînement à la lecture.

(c) La lecture extensive (Extensive reading). Elle est adaptée à des textes longs, l'objectif étant d'en extraire le sens global.

(d) La lecture intensive (Intensive reading). Elle s'applique, au contraire, sur des textes courts. Il s'agit de s'arrêter au détail pour l'analyse, la compréhension et l'assimilation du contenu. Le texte en entier n'est pas l'objectif, mais ce sont souvent les passages importants qui font l'objet de cette démarche de lecture. Il s'agit d'une lecture profonde et sans doute efficace pour les apprenants ayant un très bon niveau de langue, et faisant appel à d'autres connaissances non proprement linguistiques (culturelles, expérience vécue, intelligence, etc.).

Avant d'opter pour l'une ou l'autre de ces stratégies, il convient d'envisager les circonstances dans lesquelles l'approche du texte va être menée. S'agissant ici d'un article de journal, sélectionné à des fins pédagogiques, on peut émettre l'hypothèse que le projet ${ }^{9}$ de lecture des étudiants est de s'informer pour donner une opinion et,

\footnotetext{
${ }^{8}$ Nous ne retenons que celles qui nous paraissent utiles pour notre propos. Ces stratégies sont exposées par les auteurs, dans l'AG, sous une multiplicité de formes et de dénominations : lecture studieuse, lecture balayage, stratégie de sélection, lecture-action, lecture oralisée (Cicurel, 1991 : 1617) ; lecture écrémage, lecture survol (Tagliante, 1994 : 126-127) ; circuit court vs circuit long (Peyroutet, 1991 : 40); lecture panoramique, lecture analytique, lecture plurielle, etc. (Günday, 2010 : 196). On ne peut nier l'évidence, à la suite de Rui (2000:5), qu'une telle profusion de modalités conduit inévitablement à leur chevauchement.

${ }^{9}$ Le terme renvoie à la façon dont l'étudiant/lecteur perçoit le texte, à ses objectifs de lecture. Pour sa part, Courtillon $(2003: 45)$ envisage trois types de projets : le projet fonctionnel/pragmatique, le projet sémantique et le projet d'apprentissage. En fait, ces différents emplois font clairement apparaitre des conceptions diverses du vocable : projet d'apprentissage, projet d'usage, projet pédagogique, projet d'enseignement. Pour une analyse comparative approfondie de ces usages voir Puren (2009: 16-17).
} 
si besoin, d'interagir dans la classe. Le guidage de l'enseignant/médiateur devient essentiel, puisqu'il devra fournir les objectifs à atteindre, les activités à accomplir, et qu'il devra surtout « aider les apprenants à apprendre à comprendre un texte écrit en langue étrangère » (González Hernández, 2012: 5). Confronté au texte, l'étudiant/lecteur pourra mettre en œuvre une lecture écrémage (a) ou une lecture balayage (b), tandis que les démarches (c) et (d), sans nier leur utilité dans ce type de support, s'adapteraient mieux à d'autres types d'écrits. À la suite de Günday, la structure même du texte pragmatique (dans notre cas le texte de presse) en faciliterait sa compréhension, tant et si bien que le lecteur n'aurait pas besoin d'une analyse détaillée (Günday, 2010 : 194). Bien entendu, cette constatation, érigée en évidence, reste à nuancer. S'il paraît certain que la structure du document de presse aide à construire sa compréhension globale, il n'en reste pas moins que d'autres paramètres peuvent influer sur la construction du sens : longueur et complexité du texte, compétences de l'étudiant/lecteur, éléments paralinguistiques, projets de lecture, etc. Ainsi donc, une lecture ciblée (b) peut s'avérer utile si le but est de repérer une information ponctuelle dans le texte. De même, pour des étudiants ayant une bonne compétence en langue étrangère, une lecture intensive (d) pourrait être mise en place, si les objectifs d'apprentissage poursuivent une compréhension détaillée du texte. Dans le cadre de l'AG, la consigne joue également un rôle fondamental : celui d'orienter l'apprenant/lecteur dans la construction de la compréhension (Cicurel, 1991). Les activités qui requièrent un retour au texte contribuent en partie à atteindre ce but :

Proposer des activités de compréhension qui imposent à l'étudiant de retourner au texte, d'y rechercher des informations, est une manière d'apprendre la langue dans son fonctionnement sans que l'attention soit nécessairement portée sur la structure (Courtillon, 2003 : 44).

Établir des objectifs de lecture précis, donner des consignes pour faire agir l'apprenant, et l'aider conséquemment à comprendre le texte, font partie intégrante d'une démarche de lecture efficace. Et ce n'est que lorsque la consigne est établie que les apprenants opteront pour l'une ou l'autre des stratégies. Outre la mise en place d'activités comme: les questions/réponses, la description, l'argumentation, les échanges d'informations, etc., les projets en simulation (Cicurel, 1991:18) sont un bon moyen d'orienter le lecteur vers la réalisation d'une tâche. Et l'enseignant y ferait uniquement figure de médiateur. L'article de journal se prête aisément à cette modalité d'activité, comme nous allons le voir plus loin.

\section{Les compétences visées}

La conception de l'apprentissage exposée dans le CECR se situe dans une approche actionnelle, constituée en norme, dont s'inspirent actuellement les méthodes de langues, les enseignants et les apprenants. L'émergence de cette nouvelle perception de l'apprentissage a permis d'homogénéiser les normes existantes, et de définir les compétences en termes d'activités langagières et de savoir-faire. Le CECR 
(Conseil de l'Europe, 2001: 18) transforme les quatre compétences classiques - compréhension orale/écrite et expression orale/écrite - en quatre scénarios dits de réception, de production, d'interaction et de médiation. Aux compétences proprement linguistiques viennent s'ajouter des compétences sociolinguistiques et pragmatiques, qui vont aider « les étudiants d'aujourd'hui à être les professionnels de demain » (Gérard Locajono, 2010 : 277). Au-delà donc des compétences spécifiques propres à chaque situation d'enseignement et, corrélativement, des savoirs langagiers, les apprenants doivent acquérir des compétences générales permettant d'intégrer les aspects interculturels, des savoir-être, des savoir-faire et des savoirapprendre.

\subsection{Compétences générales}

L'enseignement/apprentissage d'une langue ne peut être entièrement conçu sans avoir recours à une prise de conscience interculturelle. Le CECR s'y réfère en ces termes :

La connaissance, la conscience et la compréhension des relations (ressemblances et différences distinctives) entre «le monde d'où l'on vient» et «le monde de la communauté cible» sont à l'origine de la prise de conscience interculturelle. Il faut souligner que la prise de conscience interculturelle inclut la conscience de la diversité régionale et sociale des deux mondes. [...] Outre la connaissance objective, la conscience interculturelle englobe la conscience de la manière dont chaque communauté apparaît dans l'optique de l'autre, souvent sous la forme de stéréotypes nationaux (Conseil de l'Europe, $2001: 83$ ).

Le Cadre reconnaît donc l'interculturalité comme une compétence nécessaire au programme d'enseignement. On exige de l'apprenant qu'il sache non seulement intégrer les connaissances nouvelles de la communauté française, mais aussi modifier ses connaissances antérieures en allant au-delà des stéréotypes. Par exemple, la construction du sens de l'article de journal sera vraisemblablement facilitée, si l'apprenant/lecteur identifie, de prime abord, les réalités culturelles qui l'intègrent, même si le sujet en lui-même ne lui est pas suffisamment proche. Ainsi donc, dans une première approche du texte, l'apprenant espagnol devra, le cas échéant, faire appel à ses connaissances sur la culture française, et, si possible, sur la réalité politico-économique du pays. Outre l'Espagne (langue d'origine) et la France (langue cible), une troisième dimension culturelle émerge avec l'apparition du Maroc, pays appartenant à l'espace francophone. L'enseignant tentera de guider les étudiants en leur faisant prendre conscience de ces trois réalités culturelles. Ils pourront alors recourir à une lecture écrémage, et, bien évidemment, aux éléments paratextuels : dans notre article, la typographie, notamment les caractères gras des intertitres, une photo on ne peut plus illustrative, et sa légende à gauche permettent de mettre en contexte le document, et favorisent conséquemment « une meilleure anticipation $d u$ sens, la mise sur pied d'hypothèses, l'activation de connaissances déjà acquises » (Cicurel, 1991: 39). L'apprenant/lecteur aura ainsi mobilisé ses connaissances à propos de Sarkozy et de Mohammed VI, ex-président de la République française (président lors de la parution de l'article) et monarque actuel du 
Maroc respectivement. Pareillement, il aura activé ses savoirs sur la ville de Marrakech. La portée de cette compétence sur la construction de la compréhension va dépendre uniquement de chaque individu, de ses savoirs préalables en rapport avec le contenu du texte, et surtout de sa capacité à les activer. Cette approche de lecture sommet-base (Cicurel, 1991) devant être complétée par le modèle base-sommet (Cicurel, 1991) dans lequel les compétences linguistiques du sujet lisant facilitent l'accès au sens.

\subsection{Compétences linguistiques}

L'analyse de la construction du sens ne peut être entièrement menée, sans l'activation des compétences linguistiques de base que l'apprenant/lecteur est censé avoir. Ces compétences correspondent, essentiellement, aux opérations de réception et de production signalées dans le Cadre (Conseil de l'Europe, 2001), bien qu'elles contribuent simultanément à l'aboutissement des processus d'interaction et de médiation. Afin de faciliter l'étape de lecture, et de favoriser la compréhension du texte, trois composantes concernant la réception textuelle semblent, à nos yeux, importantes :

a) La composante sémantique et lexicale. La construction du sens demande moins un effort de décodage des éléments lexicaux qu'une appréhension de leurs relations sémantiques. Savoir discerner, le cas échéant, les rapports sémantiques entre les éléments textuels est une bonne manière d'en saisir le sens. Ce qui peut aboutir à déchiffrer des mots inconnus partant de ces paramètres : repérage d'éléments synonymes, homonymes, antonymes, d'éléments de dérivation, éléments connotatifs, classification par champs sémantiques, identification de collocations. Bien évidemment, les compétences diffèrent d'un apprenant à l'autre, et le résultat de lecture sera, lui aussi, sensiblement différent. Or, dans un cas comme dans l'autre, il importe de discerner, au moins, le lexique courant et le lexique spécialisé.

b) La composante grammaticale ou morphosyntaxique. La capacité de compréhension augmente proportionnellement à l'identification des structures correctes de la langue étrangère apprise : phrases bien formées, correction grammaticale, etc.

c) La composante phonétique ou phonologique. L'aptitude à connaître les phonèmes de la langue étrangère, à en percevoir les aspects prosodiques et expressifs pourra, certes, aider l'étudiant à faire une lecture efficace, mais c'est, en particulier, dans l'activité de production que cette dimension se révèle plus efficace. En effet, une lecture à haute voix pour la classe sera doublement profitable si le lecteur a une bonne prononciation, s'il marque des pauses ou des accents d'insistance, même dans le cas où des fautes grammaticales se glisseraient. Ainsi, le décodage textuel et la construction de la compréhension seraient visiblement 
facilités pour les apprenants récepteurs, qui n'auraient plus qu'à interpréter le message.

Mais, au-delà des compétences linguistiques, les compétences sociolinguistique et pragmatique peuvent aussi être prises en considération. Dans l'optique de notre démarche pédagogique, ne sont traitées ici que les compétences pragmatiques.

\subsection{Compétences pragmatiques}

La mise en œuvre de la compétence pragmatique est essentielle dans les opérations de réception comme dans celles de production. Ce sont les compétences discursive, fonctionnelle et de conception schématique (Conseil de l'Europe, 2001: 96) qui sont visées ici. Relativement à la compétence discursive et fonctionnelle, elles sont liées respectivement à la structuration du texte ou du discours en termes de cohésion, de cohérence, voire de développement thématique, et à son utilité fonctionnelle : description, narration, argumentation, etc. Dans le cadre de l'AG, l'apprenant/lecteur peut aborder le texte en en identifiant le genre (article de journal) et la fonctionnalité (texte informatif), et il peut même interpréter ou résumer ce texte, l'important étant de savoir transmettre l'information de façon correcte. Pour les niveaux supérieurs, argumenter ou critiquer en produisant des énoncés corrects peuvent être des opérations importantes. Dans tout processus de réception et de production, la capacité d'identifier et d'utiliser respectivement les articulateurs discursifs (cause, conséquence, concession, etc.), ainsi que la connaissance de la structure textuelle et informationnelle du texte à lire (organisation logique, cohérence, cohésion, plan) sont des compétences qui deviennent de plus en plus importantes aux niveaux intermédiaires ou avancés.

Quant à la compétence de conception schématique, elle est reliée aux échanges verbaux, l'apprenant devant être capable de mener une conversation fluide avec son interlocuteur, en évitant les vides, en reformulant au besoin, en lui répondant le cas échéant. Bien que le texte sélectionné ne suive pas le schéma général des interactions, des activités enrichissantes de lecture interactive sont susceptibles d'être mises en place : question/réponse, accord/désaccord, etc.

Se pose ainsi la question de savoir quelles compétences, jugées prioritaires, l'apprenant/lecteur doit-il mettre en place dans notre modèle pédagogique de lecture. Autrement dit, au cours de l'opération de réception textuelle, faut-il mettre audevant de la scène la forme ou le sens ? La composante linguistique ou la composante pragmatique ? Ou les deux à la fois ? Ou bien, convient-il de focaliser l'attention sur la compétence interculturelle ? Ce qui reste clair c'est que compétences et objectifs vont de pair. Il importe donc de développer chez l'apprenant/lecteur l'une ou l'autre de ces compétences ou les trois, suivant les objectifs visés (cf. 4 cidessous) et le type de texte à aborder, l'article de journal dans notre cas. Bien évidemment, il est quand-même souhaitable de privilégier certaines compétences par rapport à d'autres, en vertu du niveau et des besoins des apprenants. Mais il ne s'agit ici que de priorités, voire même d'options personnelles, dans la mesure où un équilibre entre ces composantes tend à être recherché (Conseil de l'Europe, 2001). 
Pour notre part, et sans qu'il soit question de pencher fortement pour telle ou telle compétence, nous sommes amenée à faire un choix par ordre de priorité :

a) Pour les niveaux inférieurs (A1 à $\mathrm{B} 1$ ), les compétences générales s'avèrent un atout essentiel dans la construction du sens, puisqu'elles peuvent favoriser l'anticipation d'hypothèses sur le contenu textuel. Opération qui ne peut aboutir, en général, sans l'activation des compétences linguistiques élémentaires. Ces deux composantes sont donc, à nos yeux, importantes, si on souhaite mener une démarche de lecture efficace. Les compétences pragmatiques pouvant, elles, être acquises ultérieurement.

b) Pour les niveaux supérieurs (B2 à C2) : l'équilibre des trois compétences est souhaitable. Les apprenants ont déjà acquis une maîtrise de la composante linguistique, mais un lexique pointu, des sigles inconnus propres à la langue spécifique ou des structures grammaticales complexes peuvent être travaillés. Avec des moyens linguistiques plus poussés, les lecteurs peuvent activer leurs compétences pragmatiques, et s'attarder sur l'organisation textuelle, en affinant ainsi l'analyse. En conséquence, non seulement la lecture est-elle plus profitable et efficace, mais en plus les échanges sont plus enrichissants.

Nous allons présenter, dans ce qui suit, notre démarche de lecture à des fins didactiques, en y introduisant des activités exploitables auprès d'étudiants de FLE/FOS.

\section{Démarche de lecture d'un texte de presse: les activités de classe}

Nous proposons, dans la présente section, d'exploiter un document en ligne ${ }^{10}$ (cf. Fig.1) extrait du site lepetitjournal.com ${ }^{11}$ (cf. Fig.2) :

10 Le texte peut être lu en ligne dans son intégralité sur le site : http://www.lepetitjournal.com/marrakech/a-la-une-marrakech/72305-visite-de-sarkozy-un-premiertgv-frans-au-maroc-en-2013.html [Dernier accès le 7 novembre 2012].

${ }^{11}$ Le média des Français et Francophones à l'étranger : http://www.lepetitjournal.com/. 


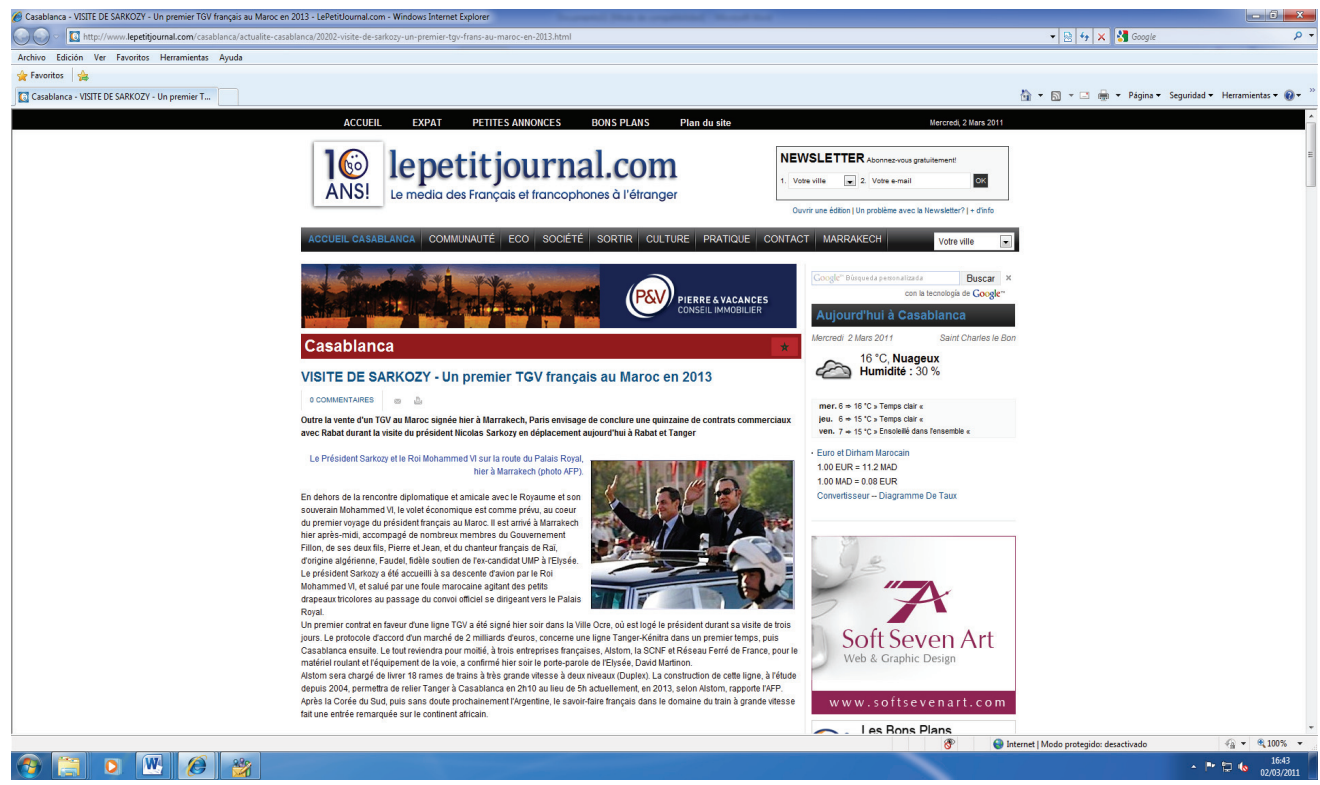

Fig.1. Article de presse en ligne

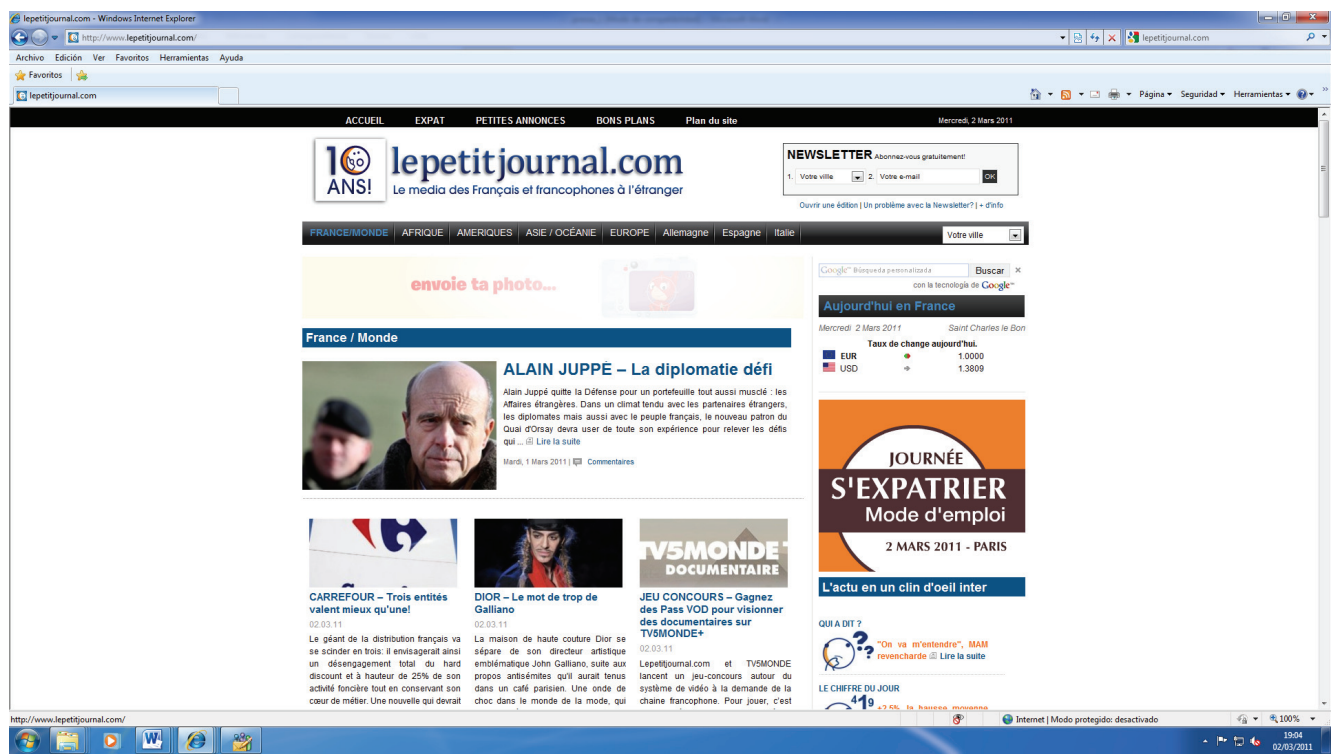

Fig.2. Portail du site lepetitjournal.com [Dernier accès le 02 mars 2011]. 
Comme nous l'avons signalé précédemment, notre choix s'est porté vers un document authentique sélectionné à des fins pédagogiques (cf. n.4). Parmi l'éventail des domaines d'information, le secteur économique nous a semblé préférable, en vue de son exploitation possible dans un cours de FLE/FOS. Pour le FLE —avec les réserves et les contraintes émises plus haut (cf. n.6) - en raison du fait que la thématique n'impose, en général, ni de vocabulaire hautement sélectif, propre à un domaine professionnel donné, ni de syntaxe lourde. Pour le FOS, contrairement à une formation plus générale, le choix du domaine en question s'explique par la présence discrète d'un lexique spécialisé qui cohabite avec un lexique standard - où mots et sigles s'entremêlent- et par la nature même de l'information apportée. En effet, relativement à sa thématique, le texte aborde la visite du président français au Maroc pour la conclusion d'un contrat commercial concernant la vente du premier TGV (Train à Grande Vitesse) français dans ce pays. On y trouve les avantages économiques dérivés de cet accord, ainsi que la perspective d'autres contrats commerciaux susceptibles d'être prochainement conclus.

Les éléments paratextuels - titre, sous-titre et illustration - annoncent ce continu informationnel, et anticipent l'événement (Mouillaud \& Tétu, 1989). Dans le cas qui nous occupe, il s'agit du voyage de Sarkozy, ancien Président de la République Française, au Maroc. Le texte est, lui-même, organisé en rubriques ou «sousensembles rédactionnels imbriqués ou répartis au sein d'un même article » (Adam, 1997 : 6). En adaptant notre modèle à celui des familles événementielles (Mouillaud \& Tétu, 1989), nous pouvons en signaler au moins quatre :

1) Les faits ou l'événement-noyau (Cicurel, 1993 : 59): la signature d'un contrat en faveur d'une ligne TGV, Tanger-Kénitra dans un premier temps, Casablanca dans un deuxième temps.

2) Les avantages : un marché de 2 milliards d'euros, $50 \%$ pour trois entreprises françaises, Alstom, la SNCF (Société Nationale des Chemins de Fer) et Réseau Ferré de France.

3) Les événement futurs : la signature d'éventuels accords (dont une Frégate Européenne Multi-Missions (Fremm) destinée à la marine marocaine), et commentaires sur les avantages économiques (500 millions d'euros). Description de ces événements : possible construction d'une centrale nucléaire dans le sud du pays ; autres protocoles d'accord et de coopération en matière de formation et de justice; ouverture d'un crédit de 38 millions d'euros pour le financement du projet d'assainissement de l'agglomération de Nador (nord du pays); don de 8 millions d'euros pour le soutien d'un programme social ciblant les régions et les quartiers les plus pauvres du Maroc; prochaine signature d'accord d'une construction d'une usine Renault-Nissan (6 000 emplois) à Tanger.

4) Le contexte : les événements qui suivent la signature du contrat. On peut relever le discours du Président Sarkozy au Parlement de Rabat et au Palais-Royal, 
ainsi que les visites protocolaires à divers chantiers auxquels participent des entreprises françaises.

Dans la démarche que nous proposons, les objectifs visés peuvent être de nature différente :

a) Objectifs pragmatiques : identification d'éléments paratextuels et de nature textuelle (plan, organisation discursive, etc.).

b) Objectifs linguistiques : chiffres, sigles, lexique lié à l'économie et éléments de syntaxe.

c) Objectifs socioculturels : importance des relations diplomatiques dans la conquête d'un marché étranger. La mondialisation.

L'enseignant est libre de modeler ces objectifs, selon les besoins et le niveau des apprenants. Par exemple, il est tenu d'insister sur les rapports socioculturels si la démarche est destinée à des étudiants de FOS, alors qu'il s'attardera probablement sur la composante paratextuelle s'il est confronté à des apprenants de FLE. Et cela, dans le but d'activer leurs connaissances préalables sur le sujet, et de les faire agir.

En ce qui concerne les activités intégrées dans notre modèle pédagogique, elles ont l'avantage de pouvoir être exploitées à des niveaux de compétence différents : élémentaire (A1/A2), intermédiaire (B1/B2) ou avancé $(\mathrm{C} 1 / \mathrm{C} 2)^{12}$. En outre, elles peuvent, bien évidemment, être complétées, modifiées, appliquées à d'autres textes, transférées à d'autres situations d'enseignement, ou réutilisées à bon escient par les enseignants de FLE/FOS. Ce sont les intérêts des enseignants et ceux des apprenants, la centration sur l'étudiant et ses objectifs d'apprentissage, qui servent de base à l'élaboration des activités à l'œuvre. Elles ont été sélectionnées en vue d'un travail individuel, par paires ou en équipe, qui mobilise l'ensemble des connaissances linguistiques et extralinguistiques des apprenants. Autant dans l'étape de préélaboration que dans celle d'élaboration de ces activités, quelques remarques d'ordre général s'imposent.

Quant au texte en lui-même:

1) Les documents de lecture doivent être les plus récents possibles. L'apprenant s'habitue à les fréquenter, et acquiert des stratégies d'observation et de construction du sens.

${ }^{12}$ Les niveaux A1-A2 du cadre correspondent à l'utilisateur élémentaire, B1-B2 à l'utilisateur indépendant et C1-C2 à l'utilisateur expérimenté (Conseil de l'Europe, 2001: 25). Dans un but de simplification, nous les avons regroupés comme suit : niveau élémentaire (A1-A2), en d'autres termes débutant (A1) et faux débutant (A2) ; niveau intermédiaire (B1/B2) et niveau avancé (C1-C2). 
2) Le même document peut être utilisé à des niveaux d'apprentissage différents. C'est la tâche demandée aux apprenants qui définit le niveau, pas le texte. Ainsi, les débutants ou utilisateurs élémentaires sont sans doute capables d'identifier des éléments connus à l'intérieur d'un document inconnu (par exemple : «Quelles villes marocaines sont-elles citées dans ce document? » ou «Quelles sont les personnes célèbres que vous identifiez dans ce document?»), mais ils auraient sans doute des difficultés pour répondre à des questions relevant d'une lecture détaillée.

Quant à l'enseignant :

Il joue le rôle de médiateur pour faire intervenir les étudiants, et les inciter à l'interaction. Il s'agit de mettre en évidence ce que l'apprenant est capable de comprendre, de dire ou de faire, suivant son niveau de français. Par exemple, il peut poser des questions auxquelles il existe une ou plusieurs réponses possibles. Pour illustration, il propose la tâche: "À deux, trouvez le plus de renseignements possibles sur le TGV français » plutôt que la question : "Qu'est-ce que le TGV ? », si l'activité s'adresse à des étudiants d'un niveau intermédiaire ou avancé. Un atout majeur est que l'enseignant pourra transformer cette tâche en un projet de simulation attrayant pour les étudiants, en le colorant d'une dimension ludique (cf. n. 3$)^{13}$.

Nous présentons, à la suite, les activités ciblées dans notre démarche de lecture.

\subsection{Les activités de réception}

La compréhension de documents écrits exige avant tout une démarche de lecture efficace. Le site du cours d'initiation à la didactique du FLE en contexte syrien (désormais CIDFLE) énumère trois types de compétences censées être mises en place $^{14}$ :

1) Une compétence de base, centrée sur l'information explicite de l'écrit.

2) Une compétence intermédiaire, cherchant à reconstituer l'organisation du texte.

3) Une compétence approfondie, visant, au-delà de l'explicite textuel, l'information rendue implicite.

${ }^{13}$ À cet égard, plusieurs sites internet peuvent servir à l'élaboration de la tâche et, conséquemment, à la mise en place d'opérations de production. Il pourra être question de rechercher des informations précises, suivant une feuille de route adressée à l'apprenant/lecteur.

${ }^{14} \mathrm{http}: / /$ www.lb.refer.org/fle/cours/cours2_CE/comp_ecr/cours2_ce02.htm [Dernier accès le 23 février 2012], non paginé. 
S'il est vrai que ces trois compétences peuvent être travaillées dans les niveaux supérieurs, il est tout aussi vrai que seule la première peut être envisagée au niveau élémentaire. Le choix va dépendre des objectifs fixés et des compétences individuelles des apprenants:

\section{1) Pour l'apprenant de niveau élémentaire:}

Il importe de développer la compétence de base (CIDFLE) en centrant la lecture sur la construction de la compréhension globale. Ce qui peut se faire autant à l'aide d'éléments paratextuels (illustration, titres, sous-titres, etc.) que textuels (mots ou sigles connus, noms propres, pays, etc.). On peut suivre le processus suivant :

a) Découverte du paratexte (Étape 1). À ce niveau, la tâche doit être simple. Son but est d'aider l'étudiant à activer ses connaissances sur les personnes qui illustrent la photo. Ainsi donc, à partir de l'image et du titre, le lecteur peut être amené à interagir dans la classe, en identifiant d'abord, puis en décrivant ces personnes. De même pour les pays à l'œuvre, ce qui exige l'activation des compétences générales de l'apprenant, en particulier celles relatives à l'interculturel. De sorte qu'une des tâches demandées peut être: «Repérez les noms de personnes, des pays et/ou des villes qui apparaissent dans le titre, le sous-titre et les intertitres. Que savez-vous de ces personnes/pays/villes?».

b) Lecture globale du texte (Étape 2). Une lecture écrémage est préférentiellement demandée pour un repérage rapide de l'information. Il pourra être question de découvrir des sigles connus (TGV, PDG (Président Directeur Général) des noms de lieux ou de marques (Détroit de Gibraltar, Palais Royal, RenaultNissan), des chiffres (2 milliards d'euros, 6000 emplois, 18 rames), des dates (2003, 2004, 2013), des noms propres (Rachida Dati, Pierre et Jean). L'enseignant peut établir plusieurs tâches à partir de ces données : des questions qui exigent des réponses précises ou des projets de simulation qui demandent une recherche d'information sur Internet. Par exemple, des questions d'ordre général comme : "Qu'est-ce que le TGV? », ou des questions ciblant une information particulière: "Quelles villes marocaines repérez-vous dans ce document? » (Rabat, Casablanca, Tanger, Marrakech), "Quelles sont les personnes célèbres que vous identifiez dans le texte? » (Sarkozy, Chirac, Mohamed VI, Hassan II). Un projet de simulation pourrait être une bonne façon d'aider les étudiants à construire leur compréhension. Par exemple, «Recherchez telle ou telle information à partir du site http://www.renault.com/fr », en sachant bien qu'à ce niveau de compétence la recherche doit être aisée.

c) Étude du vocabulaire et de la syntaxe de base (Étape3). L'activation des compétences linguistiques élémentaires est requise pour l'aboutissement du processus de compréhension globale. En cas de non-identification de sigles ou mots courants, l'enseignant pourra en dévoiler le sens : SNCF, PDG, AFP (Agence 
Française de Presse). L'enseignant peut poser des questions concernant la syntaxe : «Repérez les noms ou les pronoms qui remplacent les personnes mentionnées dans le texte », «Trouvez les adjectifs décrivant ces personnes ».

d) Compréhension globale du thème (Étape 4). L'enseignant pourra évaluer le processus de réception textuelle à la fin du parcours de lecture, en particulier après la réalisation des tâches requises. Les étudiants pourront fournir, à cet effet, des productions simples : un titre, un paragraphe court résumant les idées principales, etc.

\section{2) Pour l'apprenant de niveau intermédiaire ou avancé:}

Une compétence de base et une compétence intermédiaire peuvent être activées pour l'apprenant de niveau intermédiaire. La compétence approfondie viendrait se greffer aux précédentes pour l'apprenant de niveau avancé. Les étapes sont les mêmes que celles opérant pour l'apprenant élémentaire :

a) Identification d'éléments paratextuels. À cette étape, l'apprenant identifie la photo, le titre et les sous-titres pour en extraire les pistes nécessaires à l'élaboration d'hypothèses sur le contenu ou l'événement-noyau (Cicurel, 1992). Les compétences générales pourront l'aider dans cette tâche, si l'étudiant est capable d'activer ses connaissances antérieures sur les pays ou cultures évoqués dans le texte. De sorte qu'une orientation du type: "Que savez-vous du Maroc ? » pourra être utile pour des étudiants de niveau intermédiaire. De même, un projet de simulation est préférentiellement destiné à des étudiants de niveau avancé : «Lisez les informations publiées dans les journaux français en ligne, et recherchez des événements significatifs mettant en relief les relations bilatérales entre la France et le Maroc. Puis donnez votre opinion en l'argumentant ». Ainsi, les moyens linguistiques étant moins limités, l'anticipation du contenu informationnel peut s'avérer une tâche profitable. Les étudiants peuvent interagir dans la classe, et contribuer à l'échange d'opinions. La vérification d'hypothèses aura lieu en fin de parcours.

b) Lecture silencieuse du document en vue de sa compréhension globale. Une lecture écrémage est souhaitée s'il s'agit de synthétiser l'essentiel du contenu, tandis qu'une lecture balayage est préférable si le but est de rechercher une information particulière. Dans le premier cas, l'enseignant peut poser des questions générales: «De quoi parle le texte? », «Qu'est-ce que l'Élysée ? », ou proposer la tâche : "Quels sont les événements du texte ? Comment sont-ils décrits ? ». Dans le deuxième cas, d'autres tâches requérant une information détaillée peuvent être exigées: «Quels sont les accords, signés ou prêts à l'être, entre la France et le Maroc? », ou "Quels sont les avantages de tel ou tel accord? ». À cette étape, les activités peuvent être menées en équipe pour une plus grande interaction et, corrélativement, un plus grand profit : « En vous inspirant du do- 
cument, décrivez quels sont les bénéfices d'un tel accord, puis comparez vos résultats avec les autres participants ».

c) Étude d'un vocabulaire plus large et de structures syntaxiques plus complexes. L'enseignant peut expliquer des mots spécifiques ou des expressions difficiles à appréhender, en particulier pour des étudiants de niveau intermédiaire : rames de trains, enveloppe de 500 millions d'euros, projet d'assainissement, cibler, relayer, etc. Il pourra, en même temps, donner la signification de certains sigles ou abréviations moins courants : AFP, UMP (Union pour un mouvement populaire). $\mathrm{Au}$ niveau avancé, une maîtrise du vocabulaire spécifique est nécessaire. Les apprenants sont donc tenus d'identifier le lexique relevant du domaine économique, et d'en inférer le sens le cas échéant : protocole d'accord, zone franche, contrat commercial, savoir-faire français, arc-en-ciel de protocoles. L'activité est extrapolable aux sigles et acronymes non issus de la langue commune : INDH (Initiative nationale pour le développement humain), FREMM (Frégate Européenne Multi-Missions). Il convient que les tâches demandées ciblent, en parallèle, les problèmes de syntaxe complexes: repérage des structures phrastiques, d'éléments de coordination et de subordination, de fonctions, d'éléments corrélateurs, etc.

d) Étude de l'organisation textuelle. Mise en place des compétences pragmatiques (compétence intermédiaire (CIDFLE)) au moyen d'une lecture plus profonde. Les tâches demandées portent sur l'analyse d'éléments textuels (plan, événements, etc.) et discursifs (cohérence, cohésion, actes de langage, etc.). Par exemple le projet suivant: "Identifiez les rubriques informatives dans le texte, et argumentez leur pertinence. Puis, pensez à d'autres rubriques possibles censées l'enrichir », ou "Identifiez les repères temporels dans le texte et classez-le en fonction de leur nature » (hier soir, prochainement, aujourd'hui, lundi soir, fin août, etc.).

e) Approfondissement sur la thématique abordée pour les étudiants de niveau avancé. Une lecture intensive peut être programmée à cette fin. Elle pourra déboucher sur une réflexion critique du thème principal ou d'une thématique proche. Ainsi donc, une compétence approfondie (CIDFLE) est mise en perspective, et l'analyse pragmatique se focalise sur la visée argumentative (l'intentionnalité, entre autres (Qui ? Quoi ? Où ? Quand ? Comment ? Pourquoi ?)) et la dimension énonciative, dans le cadre de laquelle peuvent être analysés les implicites discursifs, ainsi que la position du journaliste par rapport au texte. Pareillement, à ce niveau de compétence, non seulement les objectifs pragmatiques mais aussi les objectifs socioculturels sont visés. L'enseignant pourra établir le projet suivant : " Avec quels autres pays la France serait-elle susceptible de négocier des contrats commerciaux? ». Il est demandé aux apprenants de donner leur avis, en développant et en argumentant leur point de vue. Ainsi donc, les tâches ou projets mettant en évidence l'importance des échanges bilatéraux dans la conquête 
d'un marché étranger, la mondialisation, la globalisation, les relations diplomatiques, etc. ouvrent des perspectives enrichissantes sur le plan du développement de la compétence interculturelle et de l'ouverture à l'autre.

\section{En guise de conclusion}

Dans le but de développer les compétences de lecture des apprenants de FLE/FOS, et d'aider les enseignants dans cette tâche, nous avons proposé une démarche de réception textuelle inspirée de l'AG. En effet, il nous semble qu'une telle démarche rend non seulement la lecture plus fonctionnelle et agréable, mais conduit plus efficacement aussi à l'autonomisation des apprenants/lecteurs. Notre modèle didactique fournit des pistes d'exploitation d'un document de presse en ligne, qui traite d'un sujet relevant du domaine économique. La thématique envisagée permet, au moins, de rendre la lecture plus attrayante pour des étudiants de FOS, et peut-être bien de la rendre plus vivante pour les apprenants de FLE. S'agissant d'un texte authentique relativement court, sélectionné ad hoc pour éviter le cumul d'informations, il présente un atout non négligeable : le niveau de difficulté n'étant pas trop élevé, il s'adapte à tous publics, des lecteurs débutants aux plus compétents, des apprenants de niveau élémentaire aux apprenants de niveau avancé.

Un survol des stratégies d'apprentissage a été mené, en particulier, celles concernant la lecture, et les stratégies susceptibles d'être déployées pour l'aborder. En nous inspirant du Cadre (Conseil de l'Europe, 2001), les compétences nécessaires au processus de réception textuelle ont été décrites, en tentant de privilégier ou d'équilibrer telle ou telle compétence selon le niveau de l'apprenant/lecteur. Nous en avons retenu trois pour notre propos : les compétences générales, linguistiques et pragmatiques. Notre démarche comprend trois objectifs possibles: les objectifs pragmatiques, linguistiques et socioculturels. Il importe que les tâches soient adaptées aux objectifs, et que les objectifs soient, eux-mêmes, fixés en fonction des intérêts de l'apprenant/lecteur, et surtout de son niveau de compétence. Donner primauté à l'un ou l'autre des objectifs, à l'une ou l'autre des compétences en dépend. Concernant les activités de réception, trois types de compétences doivent être, à nos yeux, ciblées : une compétence de base, une compétence intermédiaire et une compétence approfondie (CIDFLE). La première mise en place préférentiellement pour l'apprenant de niveau élémentaire, tandis que la troisième concernerait plutôt l'apprenant de niveau avancé. Les stratégies de lecture proposées pour la compréhension globale du texte suivent, à peu près, le même processus : étude du paratexte, lecture du texte, étude du vocabulaire et de la syntaxe, compréhension globale du sujet. À ajouter l'étude de l'organisation textuelle et un approfondissement sur le thème pour des étudiants de niveau intermédiaire et avancé respectivement.

Lors de l'étape de lecture proprement dite (Étape 2), les apprenants sont censés faire de l'écrémage ou du balayage. Dans le premier cas, les étudiants peuvent saisir l'information essentielle à travers le repérage des mots clés (sigles, chiffres, noms 
propres ou autres). Dans le deuxième cas, ils font une recherche plus précise sur un passage du texte, et peuvent même aller au-delà en activant, le cas échéant, leurs acquis et leurs connaissances sur le thème abordé. Les tâches suggérées sont illustrées sous forme de consignes, et elles répondent à une lecture interactive, l'enseignant n'étant qu'un simple médiateur dans la classe. C'est pourquoi, ce modèle de réception textuelle est censé favoriser la capacité d'autonomisation des apprenants, en leur facilitant une lecture à la fois critique et méthodique. En sachant bien que le texte n'a pas à être appréhendé dans sa totalité, et que sa compréhension globale n'exige pas d'en connaître les mots complexes. Ainsi, il se peut que l'apprenant de FLE puisse ne pas maîtriser le lexique spécifique, tout en ayant un niveau de langue acceptable, et que l'apprenant de FOS puisse saisir, quant à lui, le sens de certains mots spécialisés, tout en ayant des lacunes dans son vocabulaire de base de la langue étrangère. Il est donc important de choisir des textes qui éveillent l'intérêt des apprenants, en dosant «convenablement les éléments linguistiques inconnus et connus ${ }^{15}$, et d'éviter la démotivation en faisant varier les activités présentées. Nous espérons que la démarche de lecture proposée, issue de l'AG, pourra contribuer modestement à l'acquisition d'une compétence de lecture efficace, qui passe nécessairement par un processus d'appropriation de la compréhension textuelle.

\section{RÉFÉRENCES BIBLIOGRAPHIQUES}

Adam, J.-M., (1997) "Unités rédactionnelles et genres discursifs : cadre général pour une approche de la presse écrite" in Pratiques. Nº94, Juin, pp. 3-18.

Adami, H., (2009) "Les documents authentiques dans la formation des adultes migrants : pratiques pédagogiques et contraintes institutionnelles" in Mélanges CRAPEL [En ligne]. No31, pp. 159-172. Disponible sur: http://revues.univnancy2.fr/melangesCrapel/IMG/pdf/09_Adami.pdf [Dernier accès le 12 février 2012].

Agence Universitaire de la Francophonie: http://www.auf.org.

Boucher, E., (2002) "Lecture de textes à des fins académiques en L2" in La Revue de l'AQEFLS. N'23/1, pp. 58-67.

Carras, C. et al., (2007) Le français sur Objectifs Spécifiques et la classe de langue. Paris, CLE International.

Cicurel, F., (1991) Lectures interactives en langue étrangère. Paris, Hachette.

Cicurel, F., (1993) "Pré-visibilité des discours journalistiques" in Les Carnets du Cediscor. [En ligne]. $\mathrm{N}^{\mathrm{o}} 1, \quad$ pp. 55-76. Disponible sur: http://cediscor.revues.org/603 [Dernier accès le 10 octobre 2012].

\footnotetext{
${ }^{15} \mathrm{http}: / /$ www.lb.refer.org/fle/cours/cours2_CE/evaluation2/cours2_ev204.htm [Dernier accès le 23 février 2012], non paginé.
} 
Conseil de l'Europe (2001) Cadre européen commun de référence pour les langues. Paris, Didier.

Cours d'initiation à la Didactique du FLE en contexte syrien: http://www.lb.refer.org/fle/index.htm.

Courtillon, J., (2003) Élaborer un cours de FLE. Paris, Hachette.

Gérard Lojacono, F., (2010) "Créer des ressources audio pour le cours de FLE" in Çédille, Revista de Estudios Franceses [En ligne]. №, pp. 276-288, disponible sur : http://redalyc.uaemex.mx/redalyc/pdf/808/80812554016.pdf [Dernier accès le 24 février 2012].

González Hernández, A.-T., (2012) "Paramètres pour la compréhension d'un texte écrit en langue étrangère" in La Clé des Langues [En ligne]. Disponible sur : $\mathrm{http} / / / c l e . e n s-l y o n . f r / e s p a g n o l / p a r a m e t r e s-p o u r-l a-c o m p r e h e n s i o n-d-u n-t e x t e-$ ecrit-en-langue-etrangere-164559.kjsp [Dernier accès le 10 octobre 2012].

Grellet, F., (1981) Developing reading skills: a practical guide to reading comprehension exercises. Cambridge, University Press.

Günday, R., (2010) "Démarche de lecture en classe de français langue étrangère" in Synergies Turquie. No3, pp. 191-204.

Lehmann, D. \& S. Moirand (1980) "Une approche communicative de la lecture" in Le Français dans le Monde, Recherches et Applications. $\mathrm{N}^{\circ} 153$, pp. 72-79.

Moirand, S., (1979) Situations d'écrit. Paris, CLE international.

Mouillaud, M. \& J.-F. Tétu, (1989) Le journal quotidien. Lyon, Presses Universitaires de Lyon.

Peyroutet, C., (1991) La pratique de l'expression écrite. Paris, Nathan.

Puren, C., (2009) "Variations sur le thème de l'agir social en didactique des langues-cultures étrangères", in Les Langues Modernes. La revue de l'APLV [En ligne]. Disponible sur: http://www.aplv-languesmodernes.org/spip.php?article 1888 [Dernier accès le 24 février 2012].

Rui, B., (2000) "Exploration de la notion de «stratégie de lecture» en français langues étrangère et maternelle" in Acquisition et interaction en langue étrangère [En ligne]. $\mathrm{N}^{\mathrm{o}} 13$, disponible sur : http://aile.revues.org/387 [Dernier accès le 10 octobre 2012].

Tagliante, Ch., (1994) La classe de Langue. Paris, CLE International. 